\title{
Stem-loop binding protein is a multifaceted cellular regulator of HIV-1 replication
}

\author{
Ming Li, ${ }^{1,2}$ Lynne D. Tucker, ${ }^{1,2}$ John M. Asara, ${ }^{3}$ Collins K. Cheruiyot, ${ }^{1,2}$ Huafei Lu, ${ }^{1,2}$ Zhijin J. Wu, ${ }^{4}$ Michael C. Newstein, ${ }^{5}$ \\ Mark S. Dooner, ${ }^{6}$ Jennifer Friedman, ${ }^{7,8}$ Michelle A. Lally, ${ }^{2,9,10}$ and Bharat Ramratnam ${ }^{1,2,10,11,12}$ \\ 'Laboratory of Retrovirology, Division of Infectious Diseases, Department of Medicine, Warren Alpert Medical School of Brown University, Providence, Rhode Island, USA. ²ivision of Infectious Diseases, \\ Department of Medicine, The Miriam Hospital and Rhode Island Hospital, Providence, Rhode Island, USA. ${ }^{3}$ Division of Signal Transduction, Beth Israel Deaconess Medical Center and Department of Medicine, \\ and Harvard Medical School, Boston, Massachusetts, USA. ${ }^{4}$ Department of Biostatistics, Brown University, Providence, Rhode Island, USA. ${ }^{5}$ Department of Medicine, Milford Regional Medical Center, and \\ University of Massachusetts Medical School, Milford, Massachusetts, USA. ${ }^{6}$ Division of Hematology and Oncology, Department of Medicine, Rhode Island Hospital, and Warren Alpert Medical School of \\ Brown University, Providence, Rhode Island, USA. ${ }^{7}$ Center for International Health Research, Rhode Island Hospital, Providence, Rhode Island, USA. ${ }^{8}$ Department of Pediatrics, Rhode Island Hospital, and \\ Warren Alpert Medical School of Brown University, Providence, Rhode Island, USA. ${ }^{9}$ Providence Veterans Affairs Medical Center, Providence, Rhode Island, USA. ${ }^{10}$ Lifespan/Tufts/Brown Center for AIDS \\ Research, Providence, Rhode Island, USA. "COBRE Center for Cancer Research, Rhode Island Hospital, and Warren Alpert Medical School of Brown University, Providence, Rhode Island, USA. \\ ${ }^{12}$ Clinical Research Center of Lifespan, Providence, Rhode Island, USA.
}

\begin{abstract}
A rare subset of HIV-1-infected individuals is able to maintain plasma viral load (VL) at low levels without antiretroviral treatment. Identifying the mechanisms underlying this atypical response to infection may lead to therapeutic advances for treating HIV-1. Here, we developed a proteomic analysis to compare peripheral blood cell proteomes in 20 HIV-1-infected individuals who maintained either high or low VL with the aim of identifying host factors that impact HIV-1 replication. We determined that the levels of multiple histone proteins were markedly decreased in cohorts of individuals with high VL. This reduction was correlated with lower levels of stem-loop binding protein (SLBP), which is known to control histone metabolism. Depletion of cellular SLBP increased promoter engagement with the chromatin structures of the host gene high mobility group protein A1 (HMCA1) and viral long terminal repeat (LTR), which led to higher levels of HIV-1 genomic integration and proviral transcription. Further, we determined that TNF- $\alpha$ regulates expression of SLBP and observed that plasma TNF- $\alpha$ levels in HIV-1-infected individuals correlated directly with VL levels and inversely with cellular SLBP levels. Our findings identify SLBP as a potentially important cellular regulator of HIV-1, thereby establishing a link between histone metabolism, inflammation, and HIV-1 infection.
\end{abstract}

\section{Introduction}

HIV-1 becomes a newly acquired genetic locus upon integration into the human genome. Efforts to eradicate HIV-1 have been frustrated by the genomic persistence of replication-competent provirus despite promptly initiated and prolonged, potent antiretroviral therapy. In a subset of infected individuals ( $1 \%)$, viral transcription from this locus is low to absent even in activated $\mathrm{CD}^{+} \mathrm{T}$ lymphocytes that have the capacity to harbor productive infection $(1,2)$. These individuals are referred to as elite or viremic controllers, the latter having low levels of plasma viremia ( $<2,000$ copies/ml) and the former consistently maintaining levels below the limit of detection of current assays.

Individuals with atypical responses to HIV-1 infection have been intensively studied with the hope that the discovery of underlying mechanisms can be harnessed for therapeutic and preventive advances. Perhaps the most striking example is the identification of HIV-1 coreceptor CCR5. Certain individuals with mutated CCR5 are resistant to HIV-1 infection despite high-risk behaviors $(3,4)$. Pharmacologic inhibitors of CCR5 have emerged as a component

Conflict of interest: The authors have declared that no conflict of interest exists. Submitted: April 15, 2015; Accepted: May 26, 2016.

Reference information: J Clin Invest. 2016;126(8):3117-3129. doi:10.1172/JCI82360. of multidrug regimens for the treatment of infected individuals (5). More recently, elegant genome-wide association studies of controller cohorts point to immune-based mechanisms of viral control (6). For example, polymorphisms in the HLA class I locus, especially in the region of $H L A B$ and $H L A C$, are associated with slower disease progression $(7,8)$. Other immunologic factors are also likely involved, implicating arms of both adaptive and innate immunity, but with less consistent correlations with HIV-1 disease phenotype (9-11), pointing to the underlying complexity of the viral/host relationship $(12,13)$.

HIV-1 depends on the host cellular machinery to support its replication, rendering the host proteome a major determinant of viral infection. Well-known HIV-1 cellular cofactors operating at the postentry level include lens epithelium-derived growth factor (LEDGF, also known as p75; essential for HIV-1 genomic integration) $(14,15)$, positive transcription elongation factor (P-TEFb; important for HIV-1 transcription) $(16,17)$, and tetherin (which inhibits the release of progeny virions) (18). Recent genome-wide siRNA screens (19-21) and other systemic approaches $(22,23)$ have identified numerous HIV-1-related host factors, but few of the identified factors appear to be differentially expressed according to disease burden. One exception appears to be SAM domainand HD domain-containing protein 1 (SAMHD1), which depletes 
A
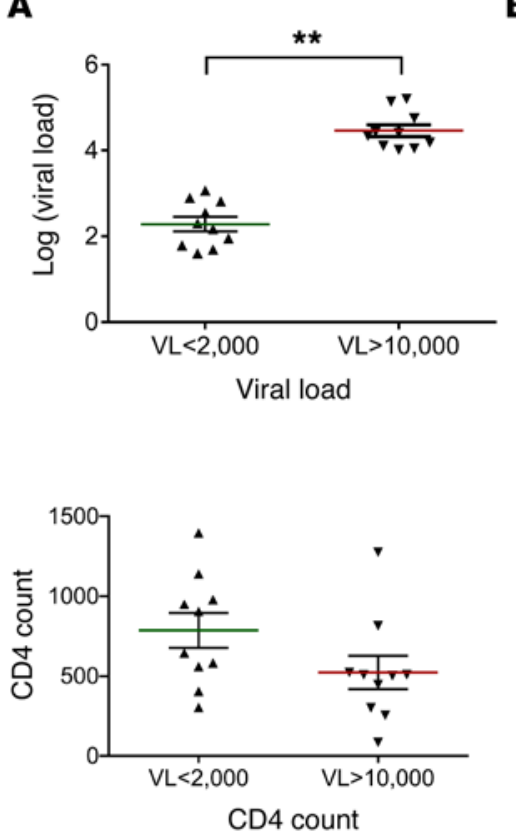

D

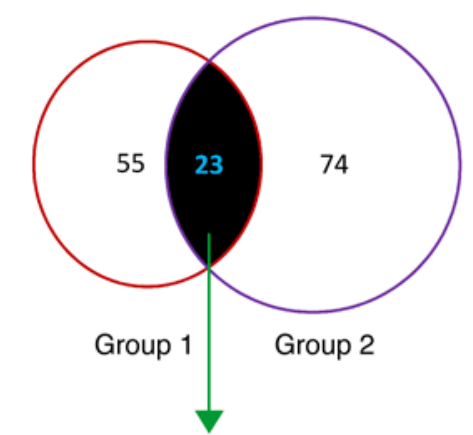

KPNA2, ICAL, SH3L1, FRIH, HP1B3, DHE3, ANXA6, CQ062, COX2, STOM, NNTM, H12, NDUB8, PGM2, S10A4, SAMH1, H1X, VINC, H2AZ, H2B1N, H4, S10A9, S10A8

B

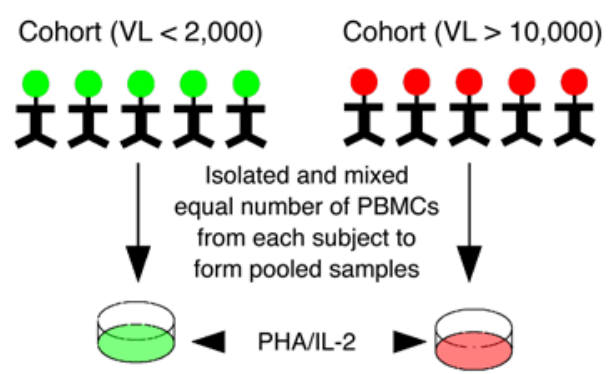

Grew in "light"

(Lys0 + Arg0) media for 7 days

Grew in "heavy"

(Lys6 + Arg10) media for 7 days

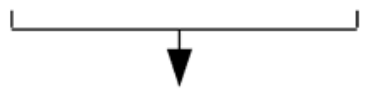

Proteins from both "light" and "heavy" pooled samples were 1:1 mixed and run on PAGE gel; bands were excised and subjected to trypsin in-gel digestion
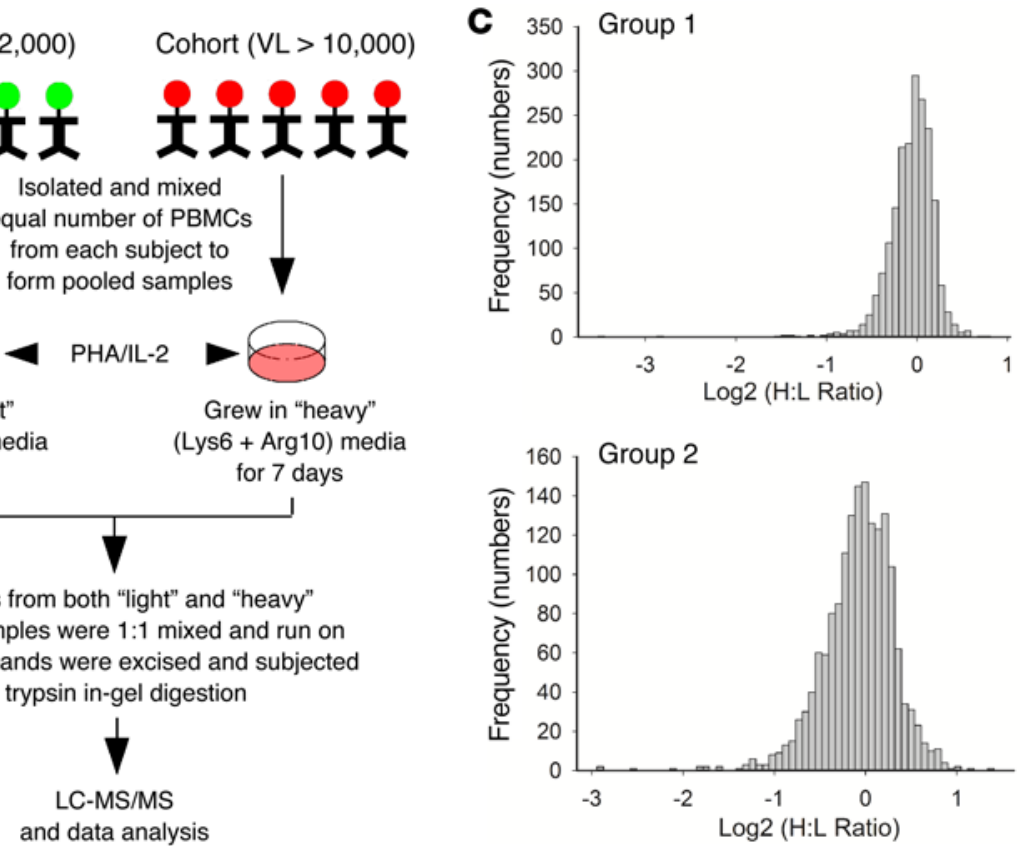

E

BP

Chromatin assembly or disassembly

DNA packaging

Nucleosome organization

Protein-DNA complex assembly

Chromatin assembly

Nucleosome assembly

\section{y}

BP

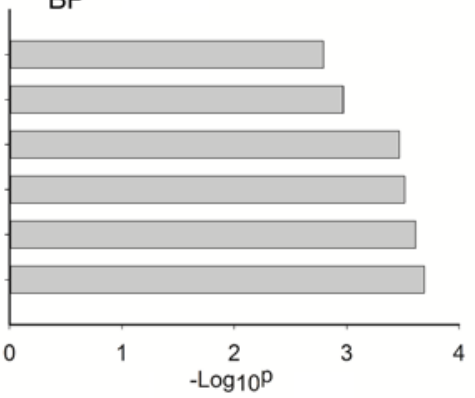

CC

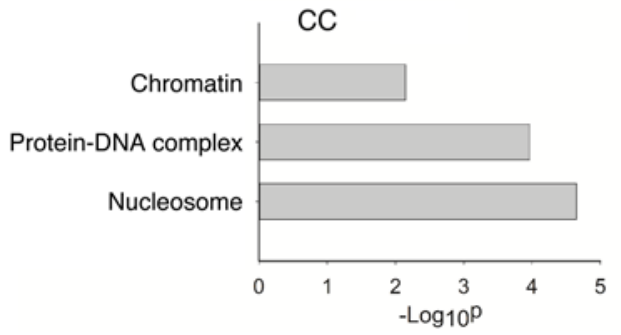

Figure 1. Proteomic characterization and bioinformatic analysis of patient samples. (A) Virologic and hematologic profile of the 20 study subjects employed in the initial proteomic screen. Top: Ten subjects had plasma VL less than 2,000 copies/ml (mean = 356 \pm 122 ). The other 10 subjects had plasma VL over $10,000$ copies $/ \mathrm{ml}$ (mean $=47,725 \pm 17,473)$. VL was statistically significant $(P<0.01)$ between the 2 groups. Bottom: The mean CD4 count between the high $(523 \pm 104)$ and the low $(787 \pm 109)$ VL group did not reach statistical significance. Mann-Whitney test; ${ }^{* *} P<0.01$; error bars represent \pm SEM. (B) Schematic diagram of the proteomic screen. In each of the 2 independent screens performed, PBMCs from 5 high-VL (>10,000) individuals were pooled and subjected to "heavy" isotopic labeling. In parallel, PBMCs from 5 low-VL $(<2,000)$ individuals received similar treatment, but with "light" media. After labeling, proteins were extracted and subjected to standard SILAC-based LC-MS/MS analysis. PHA/IL-2 (Phytohemagglutinin and Interleukin-2) (C) Histogram of SILAC ratios revealed symmetrical distribution along ratio $=1\left(\log _{2}=0\right)$ trend line for both groups. The $\log _{2}$ transformed ratios were grouped into ratio bins, and the $y$-axis showed the relative number of detected ratios per bin. (D) Differentially expressed $(P<0.05)$ candidates from the proteomic screen. Fifty-five candidates emerged from group 1, while group 2 yielded 74 candidates. Among them, 23 candidates, shared by both groups, were subjected to further analysis. (E) Statistically significant $(P<0.05)$ overrepresentation of biologic processes (BP) and cellular components (CC) based on DAVID analysis of the 23 candidates (Supplemental Table 5). There was no significant overrepresentation of molecular function.

the pool of nucleotides available for viral cDNA synthesis, thereby acting to inhibit viral replication (24). SAMHD1 is expressed at higher levels in a subset of elite controllers (25). Here, we used a reverse proteomic strategy to identify host factors that were differentially expressed according to HIV-1 plasma burden.

\section{Results}

Supplemental Results are available online with this article; doi:10.1172/JCI82360DS1.

Proteomic characterization of patient samples. We began by studying individuals with chronic HIV-1 infection who were not 
A

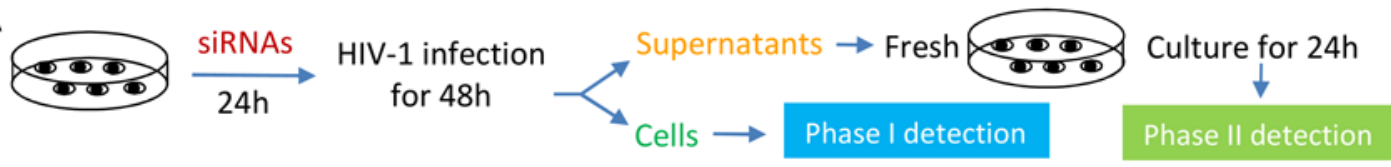

B

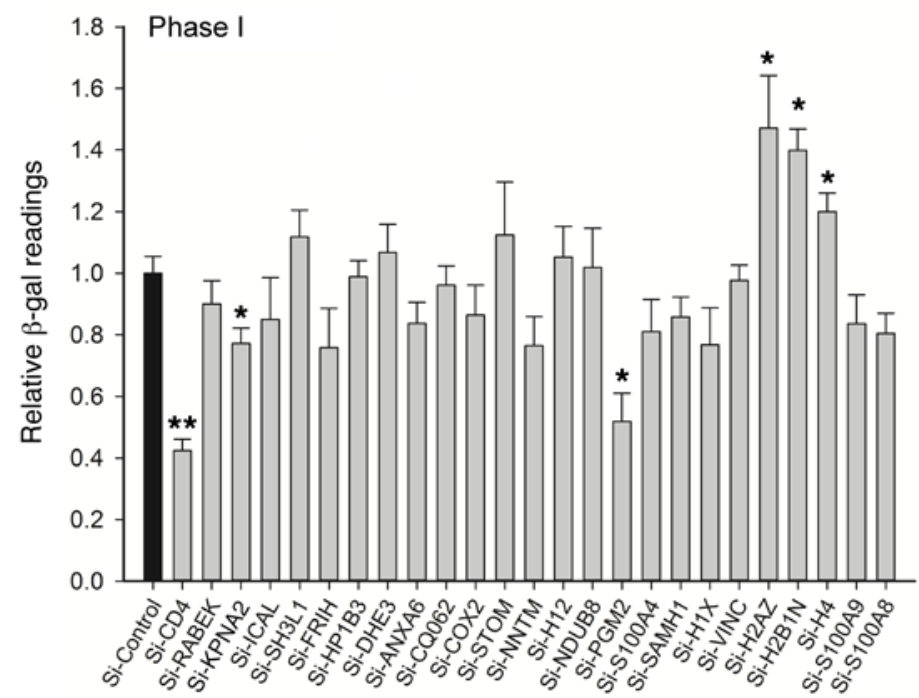

C

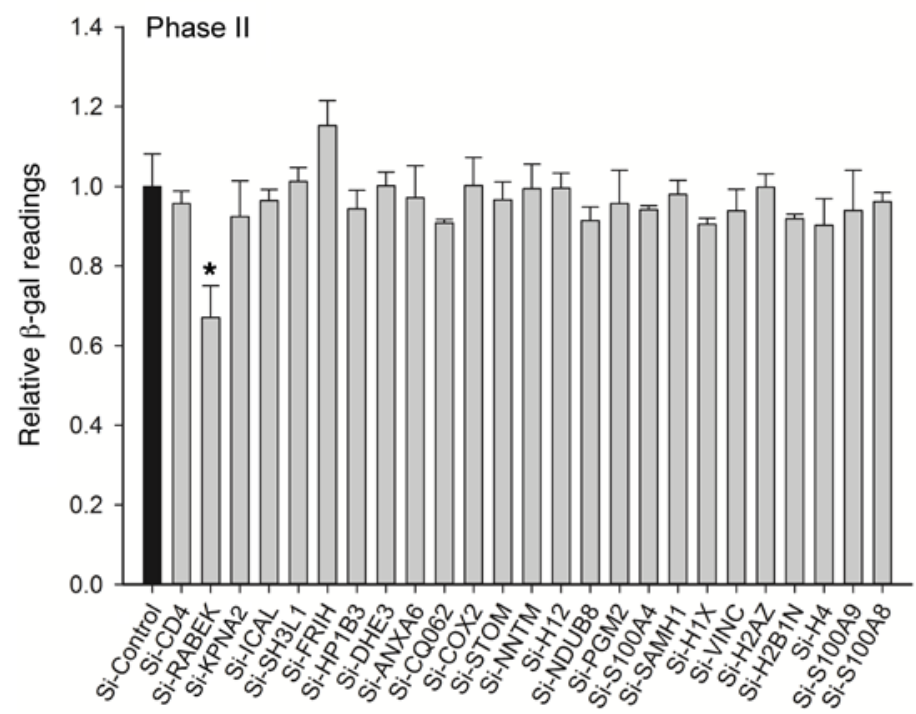

Figure 2. Identification of HIV-1-related host factors by a 2-phase $\boldsymbol{\beta}$-gal reporter assay. (A) Schematic representation of siRNA screen to identify potential roles of the 23 host candidates in the HIV-1 life cycle. siRNAs against each candidate were transfected into TZM-bl cells. HIV-1 NL4-3 $_{\text {Was }}$ added at 24 hours after transfection. Forty-eight hours after infection, cells were subjected to phase I $\beta$-gal assay. For phase II, the culture supernatant was HIV-1 p24-adjusted and added to fresh TZM-bl cells. Twenty-four hours after the addition of supernatant, the fresh cells were subjected to $\beta$-gal quantification. (B) Phase I $\beta$-gal assay results revealed that depletion of 5 of 23 proteins (KPNA2, PGM2, H2AZ, H2B1N, and H4) led to a significant change in reporter activity. (C) No candidates impacted phase II $\beta$-gal assay results. In both phase I and II assays, nontarget siRNA control (negative control), siRNA against CD4 (positive control for phase I), and RABEK (positive control for phase II) were also used. For each candidate, analyses were performed in 3 biologic replicates, each associated with technical triplicate measurements. Student's $t$ test; ${ }^{*} P<0.05$, ${ }^{* *} P<0.01$; error bars represent \pm SD.

receiving antiretroviral medication. We recruited 10 subjects with viral load (VL) less than 2,000 copies/ml (VL low) and 10 subjects with viral load greater than 10,000 copies/ml ( $\mathrm{VL}^{\text {high }}$ ) (Figure 1A and Supplemental Table 1). We randomly divided the subjects into 2 groups (each containing 5 blindly chosen individuals with high and low VL) and initially used a cohort-based analytic approach to minimize interindividual proteomic variations.

Using techniques based on Stable Isotope Labeling by Amino acids in Cell culture (SILAC) (Figure 1B), we compared the pro- teomes of the cohorts according to VL category. A total of 3,068 and 3,190 unique proteins were identified (Supplemental Table 2) from the 2 groups, respectively. Before in-depth analysis, we plotted $\log _{2}$ transformed "heavy"/ "light" ratios of all proteins from the 2 groups. The normal distribution of protein ratios indicated that our experimental techniques did not produce bias (Figure 1C). Significantly up- and downregulated protein candidates were identified by first calculating the cutoff values for the first $(>1.61$ and $<0.58)$ and second groups of data $(>2.13$ and $<0.43)$ (Supplemental 
A Low VL High VL

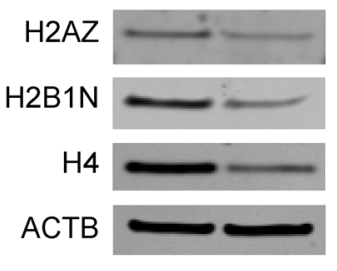

Group 1

C
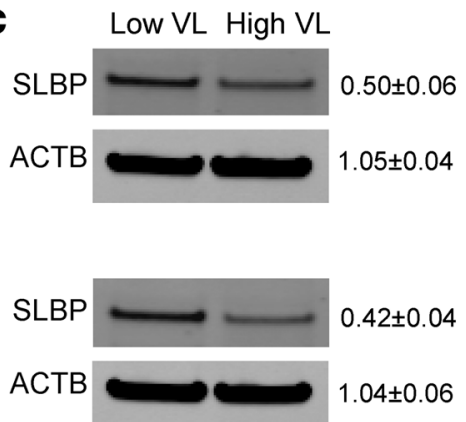

$0.46 \pm 0.13$

$0.36 \pm 0.09$

$0.31 \pm 0.05$

$1.02 \pm 0.03$
Low VL High VL

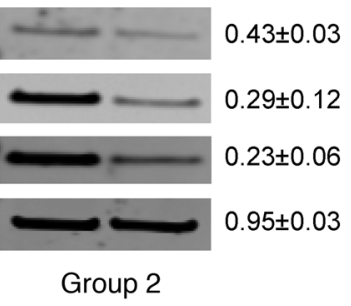

D

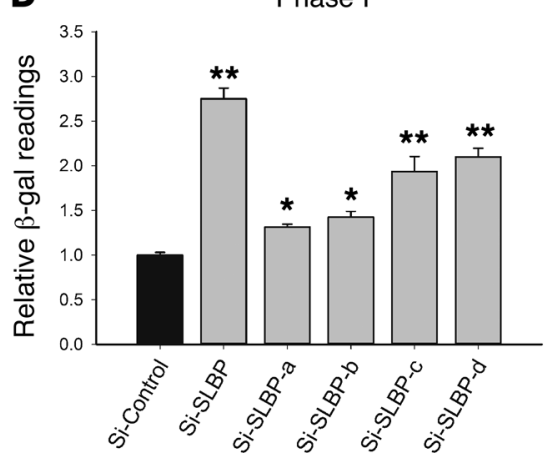

Group 1

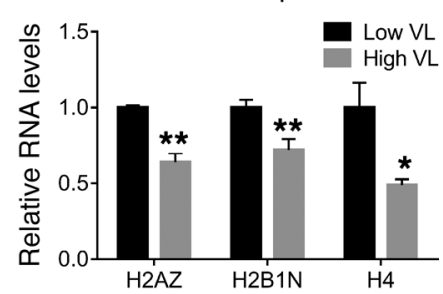

Group 2

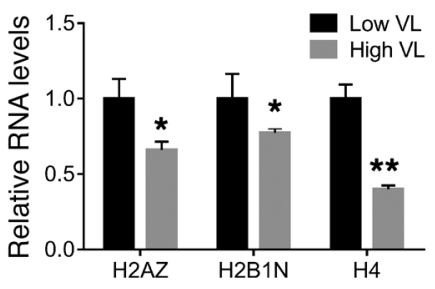

E

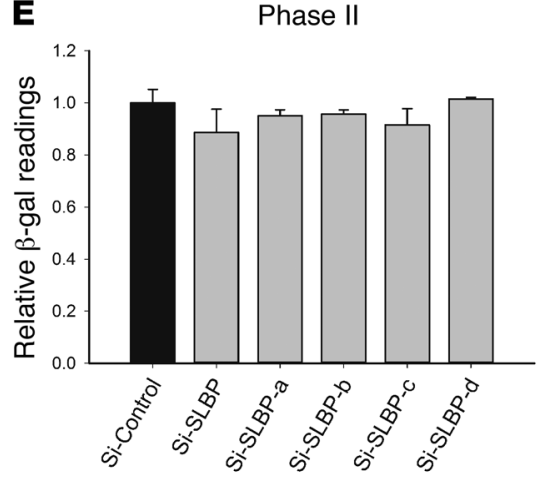

Figure 3. Histone-related stem-loop binding protein serves as an HIV-1 host factor. (A) SILAC-MS data were validated by conventional Western blots. Similar to SILAC-MS results, densitometric analysis of the 3 identified histones (H2AZ, H2B1N, and H4) revealed decreased expression in the high-VL cohort in both groups of subjects. $\beta$-Actin (ACTB) was used as a loading control. The fold changes in protein levels are noted on the right of their respective gels. (B) RNA levels of the 3 histones (H2AZ, H2B1N, H4) were significantly underexpressed (30\%-55\%) in pooled VL high samples from both groups. (C) Stem-loop binding protein (SLBP), a master regulator of histones, was underexpressed ( $50 \%$ by densitometric analysis) in pooled VL high samples in both groups. (D) SLBP depletion by SMARTpool siRNA ("SLBP") or individual siRNA ("SLBP-a"-"SLBP-d") led to higher (1.4- to 2.7-fold) $\beta$-gal readings in phase I ( $n=3$ biologic replicates). (E) SLBP depletion had no significant effects on phase II ( $n=3$ biologic replicates). The SLBP $\beta$-gal results were similar to those obtained from individual histone depletion. Student's $t$ test; ${ }^{*} P<0.05,{ }^{* *} P<0.01$; error bars represent \pm SD.

Table 3). The stringency of identification was increased by requiring that each protein associated with at least 2 unique peptides. Using these filters, a total of 55 and 74 differentially expressed protein candidates were assigned to the 2 groups, respectively (Supplemental Table 4). We merged these independent data and identified a total of 23 proteins that were shared by both groups (Figure 1D and Supplemental Table 5). Of these 23 candidates, Database for Annotation, Visualization and Integrated Discovery (DAVID) analyses revealed significant enrichment $(P<0.05)$ of nucleosome assembly-related biologic processes and nucleosome or chromatin cellular components (Figure 1E and Supplemental Table 6).

Identification of HIV-1-related host factors. The first indication that our experimental methodology was capable of identifying bona fide HIV-1-related host factors came from mining the HIV-1 Human Interaction Database (26). Of the 23 identified proteins, 8 had been previously associated with HIV-1 (Supplemental Table 5). For example, the HIV-1 restriction factor SAMHD1 (27) was expressed at lower levels in $\mathrm{VL}^{\text {high }}$. In contrast, karyopherin subunit $\alpha 2$ (KPNA2), which facilities the nuclear entry of the viral preintegration complex (28), was overexpressed in VL high . Vinculin (29), ferritin (30), and 4 histones, histone H12 (31), histone H2AZ (32), histone H2B1N (33), and histone H4 (34), were all underexpressed in $\mathrm{VL}^{\text {high }}$. To determine which of the candidate proteins had a direct effect on the HIV-1 life cycle, we used a 2-phase reporter assay that allowed us to assess the impact of RNAi-mediated depletion of each candidate protein on HIV-1 replication. The reporter assay (Figure
2A) comprised TZM-bl cells with a stably integrated HIV-1 long terminal repeat (LTR) upstream of the indicator $\beta$-gal. Viral challenge leads to HIV-1 tat-mediated engagement of the LTR, resulting in increased $\beta$-gal activity, thereby broadly assessing the effect of biologic interventions on steps of the viral life cycle from cellular entry to genomic integration and transcription (phase I). We used RNAi to deplete each of the 23 identified proteins in TZM-bl cells. The SMARTpool siRNA treatments typically achieved approximately $80 \%$ depletion efficacy on most of the candidates (Supplemental Figure 1). RNAi treatments had no significant effect on cell survival or proliferation (Supplemental Figure 2). As seen in Figure 2B, of the 23 candidates, depletion of 5 host proteins led to significant phase I effects $(P<0.05)$. Among them, the depletion of KPNA2 and phosphoglucomutase-2 (PGM2) led to approximately $20 \%$ $(P<0.05)$ and $45 \%(P<0.05)$ lower $\beta$-gal activity, respectively. Notably, depletion of all candidate histones except H1X and H12 led to between $20 \%$ and $45 \%(P<0.05)$ increase in $\beta$-gal activity. We confirmed these results using 4 independent siRNAs for each of the 5 significant hits (KPNA2, PGM2, H2AZ, H2B1N, and H4) (Supplemental Figure 3). We next addressed whether any of the candidate proteins affected viral infectivity. Culture supernatants from siRNA-treated cells were p24-adjusted and applied to new TZM-bl cells, and $\beta$-gal activity was measured (phase II). The supernatants derived from the cells with individually depleted proteins did not produce significant changes in $\beta$-gal readings in recipient cells (Figure $2 \mathrm{C}$ and Supplemental Figure 4). These results likely reflected 


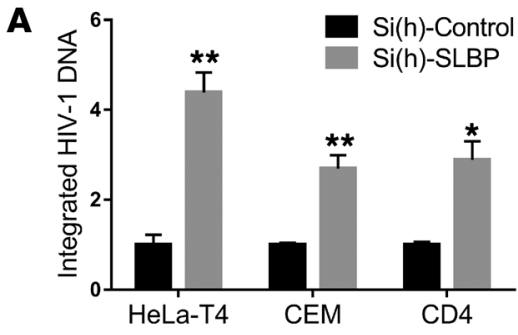

C

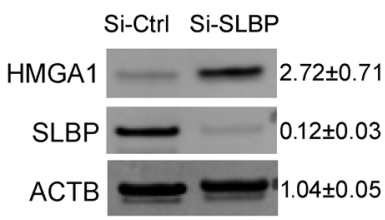

HeLa-T4

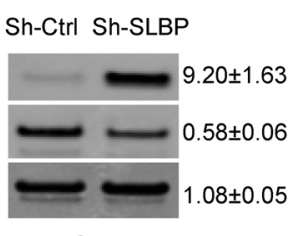

CEM
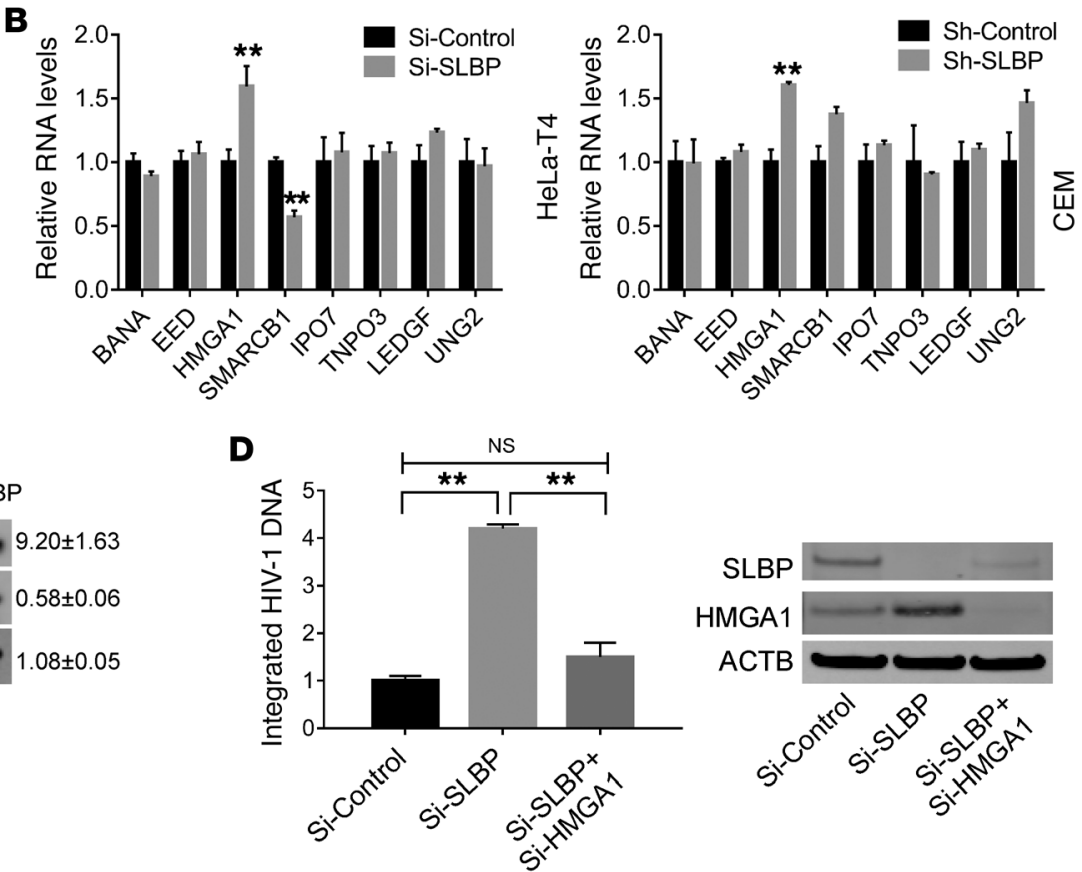

E

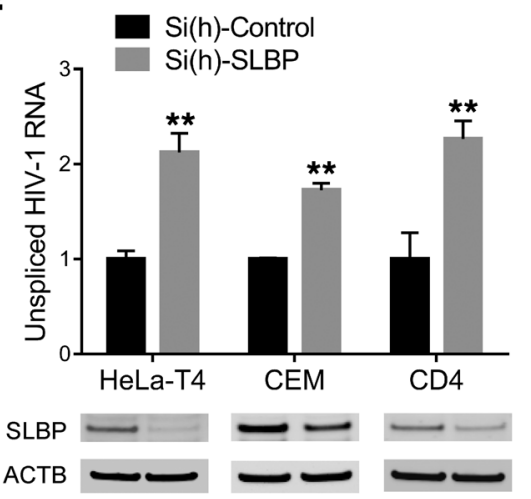

$\mathbf{F}$

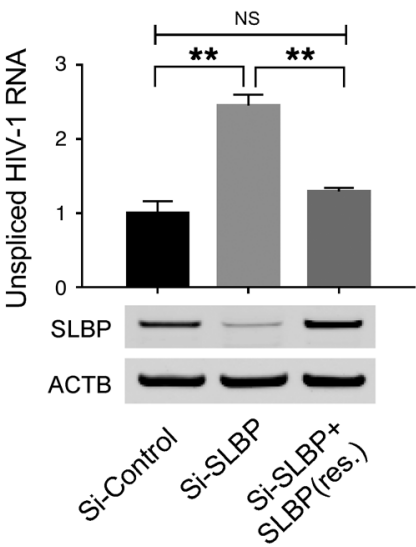

G

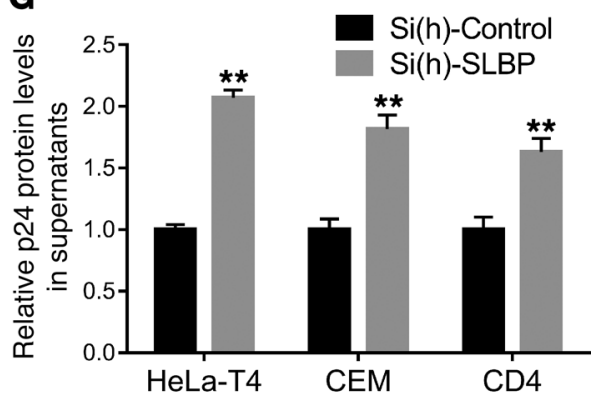

Figure 4. SLBP depletion led to higher levels of HIV-1 integration and replication. (A) SLBP depletion in HeLa-T4, CEM, and primary CD4+ T Iymphocytes

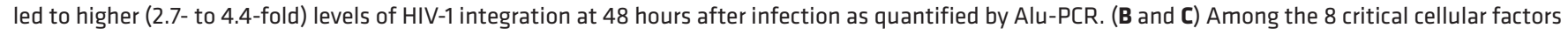
(BANF1, EED, HMGA1, SMARCB1, IP07, TNP03, LEDGF, and UNG2) involved in HIV-1 integration, SLBP depletion led to increases in HMGA1 mRNA (B) and protein (C) expression. The fold changes in protein levels were quantified by densitometric analysis and are noted on the right of gels. (D) The impact of

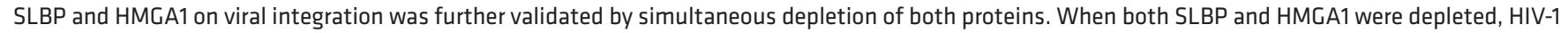
integration levels were reduced and approached control conditions. (E) SLBP depletion in HeLa-T4, CEM, and primary CD4+ T Iymphocytes led to significantly higher (1.7- to 2.3-fold) levels of unspliced HIV-1 mRNA expression at 48 hours after infection as quantified by real-time PCR. (F) The specificity of

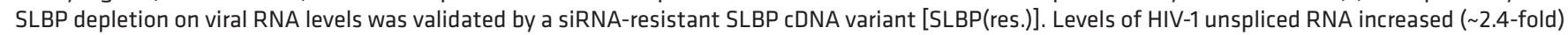
upon SLBP depletion but returned toward control levels when SLBP siRNA-treated cells expressed SLBP(res.). (G) SLBP depletion in HeLa-T4, CEM, and primary CD4+ T lymphocytes led to significantly higher ( 1.6- to 2.1-fold) levels of p24 in culture supernatant at 48 hours after infection as quantified by ELISA. Western blots were used to measure and verify HMGA1 and SLBP protein levels for each experimental condition. ( $n=3$ biologic replicates.) Student's $t$ test was performed for A, B, E, and G. One-way ANOVA with post hoc Tukey's test was used for $\mathbf{D}$ and $\mathbf{F}$. ${ }^{*} P<0.05$, ${ }^{*} P<0.01$; error bars represent $\pm \mathrm{SD}$. $\mathrm{Si}(\mathrm{h})$, short interfering (hairpin) RNA.

the complexity of virion biogenesis involving the activity of multiple human proteins with high levels of redundancy (19).

Stem-loop binding protein serves as a histone-related HIV-1 host factor. Notably, among the 5 significant candidates identified in phase I, 3 were histones. We first validated the mass spectrometry-based quantifications by Western blot profiling of the original samples. As seen in Figure 3A, histone protein levels were reduced approximately $50 \%$ to $70 \%$ in the $\mathrm{VL}^{\text {high }}$ compared with the $\mathrm{VL}^{\text {low }}$ cohort. RT-PCR profiling revealed an approximately $30 \%$ to $55 \%$ $(P<0.05)$ reduction among the $\mathrm{VL}^{\text {high }}$ cohort (Figure $\left.3 \mathrm{~B}\right)$. The mechanism of simultaneous underexpression of multiple histones in $\mathrm{VL}^{\text {high }}$ was at first perplexing. Interestingly, histones share a unique feature in that their mRNAs end with a $3^{\prime}$ stem-loop structure rather than the ubiquitous poly-A tail (35). Stem-loop binding 
A

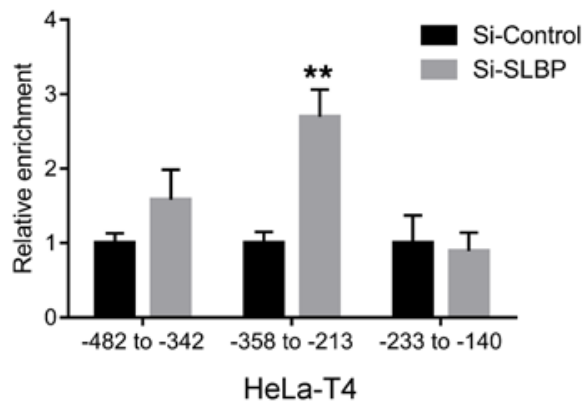

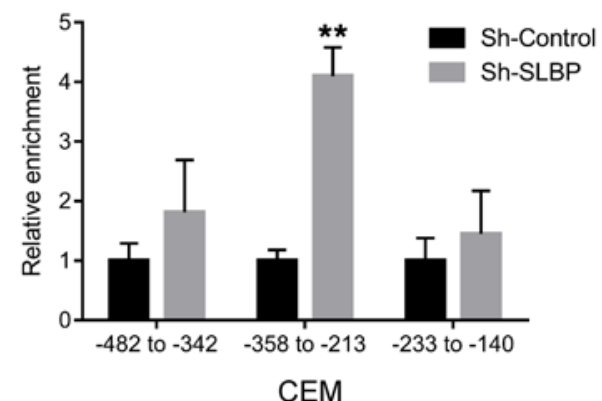

CEM

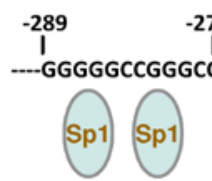

B
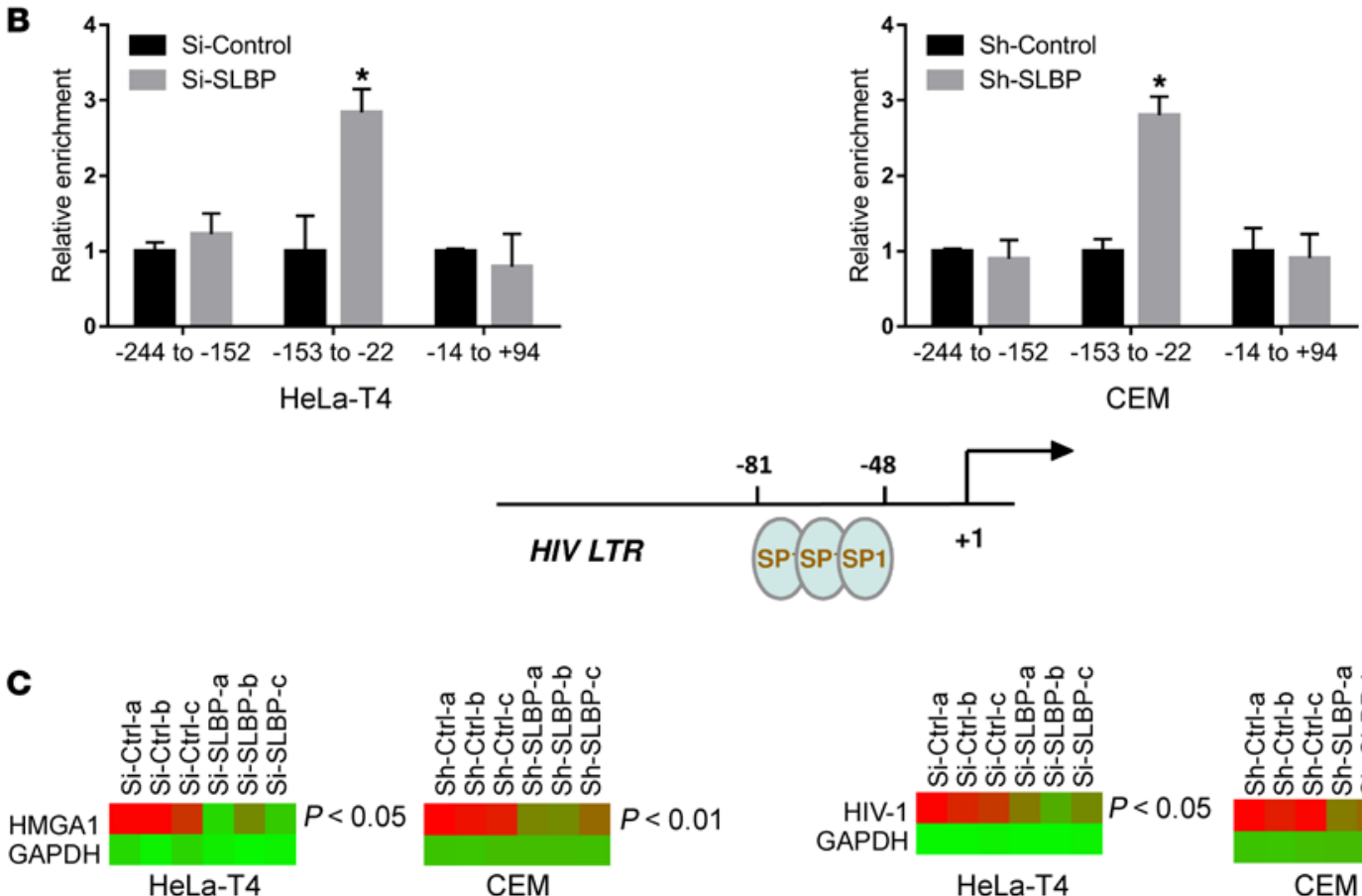

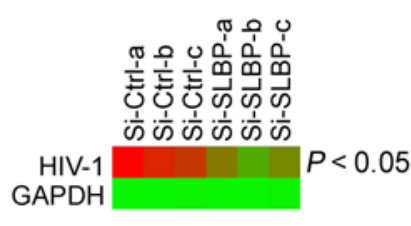

HeLa-T4

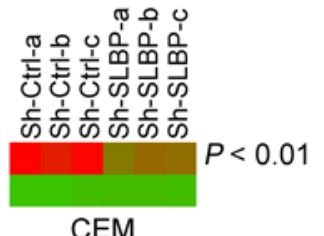

CEM

Figure 5. Impact of SLBP depletion on HMCA1 and HIV-1 promoter activity and transcription factor accessibility. (A) ChIP assay revealed that the transcription factor SP1 had higher binding affinity to specific promoter regions of HMGA1 upon SLBP depletion with relative enrichment of binding in the region -358 to $-213(P<0.01)$. This was in agreement with predicted (EpiTect ChIP; Qiagen) SP1 binding sites $(-289$ to -278$)$. (B) ChIP assay showed that the transcription factor SP1 also had higher binding affinity to specific regions of the HIV-1 promoter (or LTR). SP1 binding was enriched in the region of -153 to $-22(P<0.05)$. The bottom schematic diagram shows the known binding regions of SP1 to HIV-1 LTR (http://www.hiv.lanl.gov/). (C) SLBP depletion was associated with higher transcription factor accessibility of promoter regions of both HMGA1 and HIV-1 as defined by EpiQ assay in both HeLa-T4 cells (left panel) and CEM cells (right panel). Real-time PCR was carried out using EpiQ primers specifically designed for human HMCA1 and HIV-1, respectively, and promoter accessibility (\%) was quantified. Each heat map was based on 3 independent accessibility values from both the nontarget control and the SLBP experimental arm. Green represents high accessibility, while red represents low accessibility. GAPDH (constitutively expressed, fully accessible) was used as the control. Student's $t$ test; ${ }^{*} P<0.05,{ }^{* *} P<0.01$; error bars represent \pm SD.

protein (SLBP) is known to bind to the stem-loop structure and is involved in stabilizing mature histone mRNAs as well as regulating their transport and translation $(36,37)$. In cell culture, RNAi-mediated reduction of SLBP led to decreased expression of histone candidates at both protein and mRNA levels (Supplemental Figure 5 and summarized in Supplemental Table 7). The close correlation between histone and SLBP prompted us to profile SLBP expression in our original cohorts by Western blot; we documented approximately $50 \%$ underexpression in both $\mathrm{VL}^{\text {high }}$ groups (Figure $3 \mathrm{C}$ ). We next measured the effect of SLBP reduction on the viral life cycle using the previously described 2-stage reporter assay. As seen in Figure 3D, SLBP depletion was associated with an approximately 2.7 -fold increase $(P<0.01)$ in $\beta$-gal activity with SMARTpool siRNA and up to a 2 -fold increase $(P<0.05)$ of reporter activity when treated with individual siRNA species. SLBP siRNA treatment achieved over $80 \%$ depletion efficacy with SLBP SMARTpool and $70 \%$ to $80 \%$ with individual SLBP siRNA (Supplemental Figure 6) with no significant effect on cell survival or proliferation (Supplemental Figure 7). Similar to our work with individual histone proteins, SLBP depletion had no effect on viral infectivity. 
A

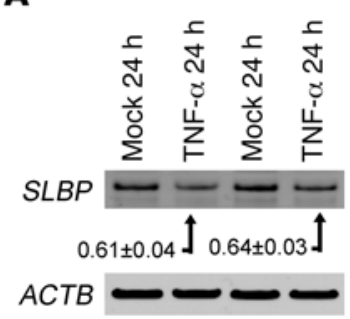

B

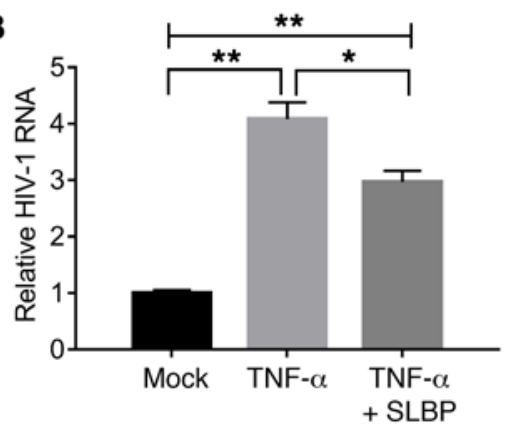

C

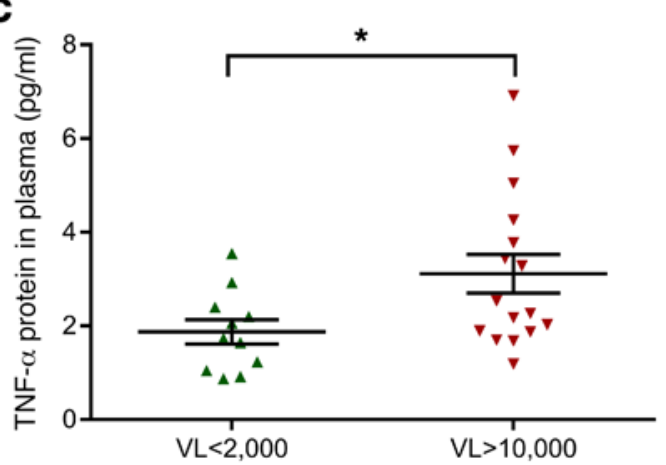

D

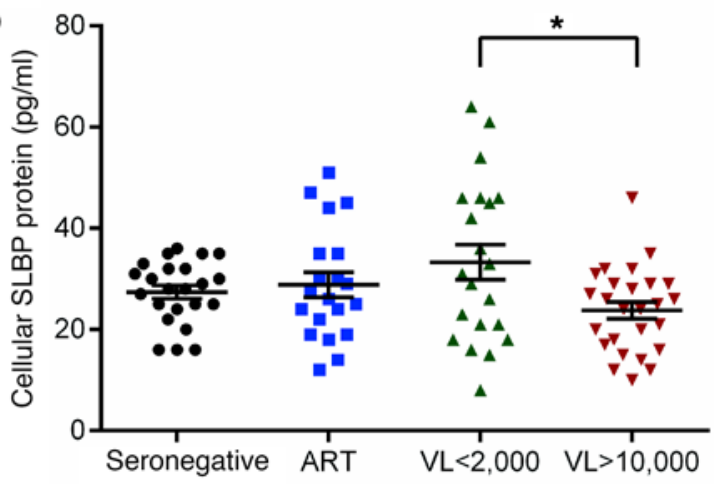

$\mathbf{E}$

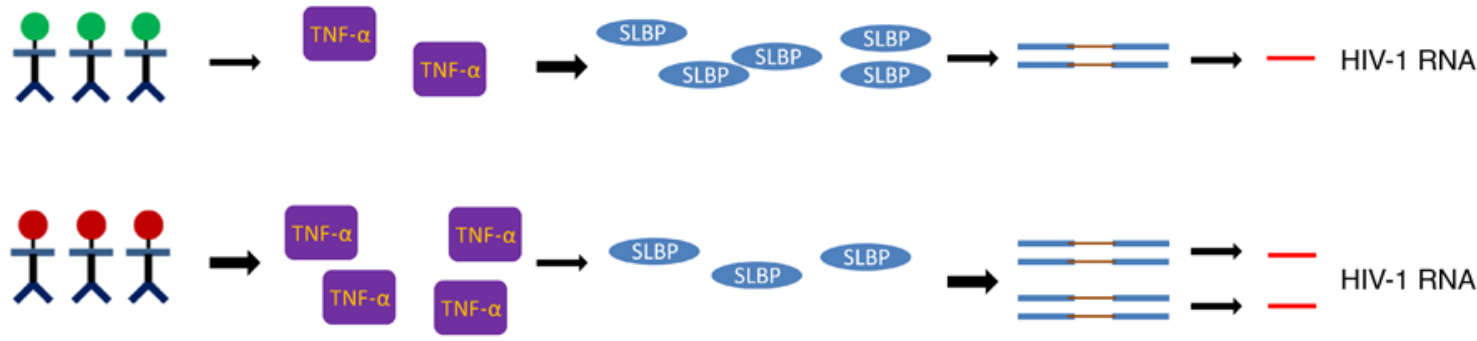

Figure 6. TNF- $\alpha$ downregulates SLBP expression. (A) In vitro TNF- $\alpha$ treatment decreased SLBP expression. CEM cells were treated with TNF- $\alpha$ $(30 \mathrm{ng} / \mathrm{ml})$. At 24 and 48 hours after treatment, SLBP protein and RNA levels were reduced by approximately $40 \%$ as quantified by Western blot (left panel) and RT-PCR (right panel), respectively. ACTB was used as the loading control ( $n=3$ biologic replicates). Student's $t$ test; ${ }^{*} P<0.05$; error bars represent \pm SD. (B) Treatment of CEM cells by TNF- $\alpha$ with subsequent HIV-1 infection led to significantly higher ( $\sim$-fold) levels of viral unspliced RNA in comparison with untreated cells or those simultaneously treated with an SLBP expression vector (TNF- $\alpha+$ SLBP). Note that superphysiologic expression of SLBP significantly decreased the HIV-1 enabling activity of TNF- $\alpha$ by $30 \%$ ( $n=3$ biologic replicates). One-way ANOVA with post hoc Tukey's test; ${ }^{*} P<0.05,{ }^{*} P<0.01$; error bars represent \pm SD. (C) Plasma levels of TNF- $\alpha$ in the original cohorts $(n=27)$ were quantified by ELISA and were significantly elevated in the high-VL group. Mann-Whitney test; ${ }^{*} P<0.05$; error bars represent \pm SEM. (D) The number of study subjects was expanded to include HIV-1-uninfected individuals $(n=23)$, those on suppressive antiretroviral therapy (ART) $(n=20)$, and additional individuals with VL less than 2,000 $(n=21)$ and VL greater than 10,000 $(n=26)$. Intracellular SLBP expression was approximately $40 \%$ higher $\left({ }^{*} P<0.05\right)$ in the low-VL versus the high-VL group. SLBP protein levels in PBMCs were quantified by ELISA for each subject. One-way ANOVA with post hoc Tukey's test; ${ }^{*} P<0.05$; error bars represent \pm SEM. (E) A proposed TNF- $\alpha / S$ LBP circuit that impacts HIV- 1 integration and transcription. TNF- $\alpha$ induced by proinflammatory stimuli decreases cellular SLBP levels, leading to enhanced HIV-1 integration and proviral transcription via an SLBP-mediated increase in viral and HMGA1 promoter accessibility.

Supernatants (p24-adjusted), derived from SLBP-depleted and HIV-1-infected cells, did not produce significant changes in $\beta$-gal readings in new recipient cells in phase II of our assay (Figure 3E). Importantly, to discount the possibility that histone and SLBP reduction was merely a consequence of the stress of cellular HIV-1 infection, we also profiled its expression in infected and uninfected primary mononuclear cells. Neither HIV-1 infection nor the culture conditions that we used impacted histone/SLBP expression levels (Supplemental Figure 8).
SLBP depletion increases HIV-1 integration and replication. Life cycle-specific assays revealed that SLBP depletion had the most marked effect on integration and transcription. We used AluPCR to quantify levels of integrated provirus and observed an approximately 4 -fold increase in HIV-1 integration levels at 48 hours after infection $(P<0.01)$ in SLBP-depleted HeLa-T4 cells. Similarly, SLBP depletion led to a significant $(P<0.01)$ increase of HIV-1 integration in lymphocytic CEM cells and primary CD $4^{+}$ $\mathrm{T}$ lymphocytes at 48 hours after infection (Figure $4 \mathrm{~A}$ ). To deter- 
mine the underlying mechanism, we surveyed a panel of 8 host factors, all known to be important for viral integration, including barrier to autointegration factor 1 (BANF1) (38), polycomb protein EED (39), high mobility group protein A1 (HMGA1) (40), SWI/ SNF-related matrix-associated actin-dependent regulator of chromatin subfamily B member 1 (SMARCB1) (41), importin-7 (IPO7) (42), transportin-3 (TNPO3) (43), LEDGF (44), and uracil-DNA glycosylase (UNG2) (45). Their RNA expression was compared between SLBP-depleted and matched control HeLa-T4 and CEM cells. Among them, only HMGA1 consistently had a significantly higher expression $(P<0.01)$ in both RNA (Figure $4 \mathrm{~B})$ and protein (Figure 4C) levels upon SLBP depletion. As a component of preintegration complexes, HMGA1 facilitates HIV-1 integration by stimulating integrase activity (40). Interestingly, HMGA1 overexpression (Supplemental Figure 9) led to an approximately 2.6-fold increase $(P<0.01)$ in HIV-1 integration level. To determine whether HMGA1 increase following SLBP depletion was mechanistically responsible for increased HIV-1 integration, we subjected cells to simultaneous SLBP and HMGA1 depletion. Compared with SLBP depletion, double depletion significantly decreased HIV-1 integration with levels approaching those in the nontargeting control (Figure 4D). Recall that our initial TZM-bl reporter assay also suggested increased proviral transcription upon SLBP depletion. This was directly borne out by a series of experiments in which we used siRNA against SLBP and found an approximately 2 -fold $(P<0.01)$ increase of unspliced HIV-1 RNA at 48 hours after infection in HeLa-T4 cells. A similar pattern of upregulation of RNA expression was also observed in SLBP-depleted CEM cells and primary CD $4^{+}$ $\mathrm{T}$ lymphocytes (Figure 4E). We further validated SLBP-mediated effects on viral replication by introducing a mutated form of SLBP (resistant to siRNA degradation but encoding functional SLBP protein; Supplemental Figure 10) into SLBP siRNA-transfected HeLa-T4 cells. As expected, provision of exogenous siRNA-resistant SLBP to SLBP-depleted cells abrogated approximately $80 \%$ $(P<0.01)$ of the effects of SLBP depletion on HIV-1 RNA increase (Figure 4F). SLBP depletion also led to a significant increase ( 1.6to 2.1-fold) in HIV-1 p24 levels in supernatants of virus-challenged HeLa-T4 and CEM cells and primary CD4 ${ }^{+} \mathrm{T}$ lymphocytes (Figure 4G). SLBP depletion had no impact on HIV-1 binding, reverse transcription, or the expression of key host factors involved in viral budding (Supplemental Figures 11-13).

SLBP depletion increases HMGA1 and HIV-1 promoter accessibility. Recent investigations have demonstrated that manipulation of histone stability impacts HIV-1 biology (46). The most direct example of this has been both in vitro and in vivo work with histone deacetylase inhibitors (HDACIs) such as suberoylanilide hydroxamic acid (47). These agents appear to have HIV-1-centric effects similar to those of SLBP depletion in that both increase cellular susceptibility to infection and transcription of integrated provirus (48). To determine whether HDACIs and SLBP depletion led to similar perturbations of histone posttranslational modifications, we quantified levels of 4 representative posttranslational modification markers (H3-ac, H3K4m3, H3K9m3, and H3K27m3) and could not find significant changes induced by SLBP depletion (Supplemental Figure 14). In addition, chromatin environments composed of abundant active regulatory elements (euchromatin) have been associated with increased levels of HIV-1 integration and transcription (49).
By performing Formaldehyde-Assisted Isolation of Regulatory Elements (FAIRE) (50) assay, we found that SLBP depletion did not significantly change the abundance of active regulatory elements, suggesting that large-scale chromatin alteration by SLBP depletion was not contributing to its HIV-1-centric effects (Supplemental Figure 15). We next explored whether SLBP depletion induced a chromatin effect(s) on a more localized, HIV-1-centric basis and altered the binding of specific HMGA1 and HIV-1 LTR transcription factors such as SP1 $(51,52)$. ChIP assays revealed significantly higher SP1 binding to the HMGA1 promoter at the region of -358 to -213 in both SLBP-depleted HeLa-T4 and CEM cells (Figure 5A). ChIP assays on SLBP-depleted and virus-challenged cells revealed an approximately 3-fold increase in SP1 binding to the HIV-1 LTR regions -153 to -22 (Figure 5B) in both SLBP-depleted HeLa-T4 and CEM cells. SLBP depletion had no effect on the SP1 expression itself, regardless of HIV-1 infection (Supplemental Figures 16 and 17). This prompted us to assess the chromatin structure and local accessibility of HMGA1 and HIV-1 promoters. As seen in Figure 5C, SLBP depletion was indeed associated with significantly $(P<0.05)$ higher accessibility of both HMGA1 and HIV-1 promoters in both HeLa-T4 and CEM cells. These results partially explained our ChIP findings in which we observed higher transcription of both HMGA1 and HIV-1 without higher levels of active regulatory elements and enhanced expression of the transcription factor SP1.

TNF- $\alpha$ downregulates SLBP expression. Our results thus far indicated that manipulations of SLBP protein levels altered HIV-1 replication dynamics. We subjected cells to a variety of stresses (e.g., activation, drug and cytokine treatment) known to render cells more susceptible to HIV- 1 infection and quantified levels of SLBP. Surprisingly, TNF- $\alpha$ treatment led to reduction of SLBP protein and RNA levels by approximately $40 \%$ (Figure 6A). Treatment of CEM cells with TNF- $\alpha$ with subsequent HIV-1 infection led to approximately 4 -fold $(P<0.01)$ increase of HIV-1 unspliced RNA levels, consistent with previous reports (51). Compared with TNF- $\alpha$-treated cells, forced supraphysiologic expression of SLBP in TNF- $\alpha$-treated cells blunted the proinflammatory effects with a significant reduction of HIV-1 unspliced RNA levels by approximately 30\% $(P<0.05)$ (Figure 6B). We next asked whether a TNF- $\alpha /$ SLBP/HIV-1 circuit was operational in vivo. We compared plasma levels of TNF- $\alpha$ in subjects with low versus high VL. As seen in Figure 6C, plasma levels of TNF- $\alpha$ were significantly higher $(P<0.05)$ in the high-VL group. Conversely, SLBP levels were approximately $40 \%(P<0.05)$ higher in the low-VL versus the high-VL group (Figure 6D). This prompted us to quantify SLBP levels in 2 additional cohorts of 43 individuals with low to undetectable levels of plasma TNF- $\alpha$ : seronegative individuals and those treated with suppressive antiretroviral medications (53). As seen in Figure 6D, SLBP levels in both of these cohorts were similar to that observed in the low-VL group, supporting the link between plasma TNF- $\alpha$ levels and intracellular SLBP protein expression.

\section{Discussion}

The genomic persistence of replication-competent HIV-1 is a substantial barrier to curing infection. While strategies aimed at achieving complete eradication of provirus are being developed and tested, most agree that this is a daunting challenge. Another approach has been to identify and therapeutically implement the components 
of a functional cure. A functional cure implies the continued existence of integrated virus but one that has been rendered nonpathogenic by immune, genetic, or pharmacologic means. HIV-1-infected individuals who are able to control viral replication without antiretroviral therapy - that is, elite controllers - are an extreme example of individuals living a functional cure. An important caveat is that even though individuals may be able to completely suppress viral replication, a fraction appears to lose viral control with time, and others appear to be at higher risk of developing illnesses associated with prolonged inflammation (54). Nevertheless, detailed studies of atypical viral resistance/control have shed light on basic issues of HIV-1 pathogenesis that have been subsequently mined for therapeutic advances (e.g., development of anti-CCR5 drugs).

Here, we set out to determine whether autonomous HIV-1 control was associated with a distinct protein signature. We found that 23 proteins were differentially expressed among individuals with high versus low VL, with histones constituting the largest class. This prompted us to focus on SLBP with its known ability to regulate histone expression. We found that SLBP exerted a multidimensional effect on HIV-1 and that SLBP is relatively underexpressed among individuals with high VL versus low VL. SLBP depletion leads to increased HIV-1 integration and viral replication. Although the effects are similar to those induced by HDACIs such as suberoylanilide hydroxamic acid, we found that acetylation did not play a role in SLBP-induced HIV-1 activation. Rather, SLBP regulates HIV-1 expression by controlling histone metabolism and affecting the accessibility of not only the HIV-1 promoter but also that of the gene encoding HMGA1 that is involved in HIV-1 integration.

Most would agree that endogenous HIV-1 control is largely immunologically based as recent studies indicate (6-11), and our data do not suggest that SLBP protein levels singularly confer controller status. Rather, our work found an unexpected circuit that links TNF- $\alpha$ to SLBP expression in vivo. This allowed us to characterize the effects of proinflammatory stimuli on the HIV-1-centric cellular scaffold. These observations provide insight into HIV-1 acquisition and disease progression. For example, sexually transmitted bacterial and viral infections (STIs) are known to increase HIV-1 susceptibility and replication by their upregulation of proinflammatory cytokines such as TNF- $\alpha$ (55). It is conceivable that STI-induced TNF- $\alpha$ may lead to decreased SLBP expression in mucosal lymphocytes, thereby increasing HIV-1 cellular susceptibility and replication (Figure 6E). A similar dynamic may also be implicated in the natural history of untreated HIV-1 disease with high levels of inflammation ensuring the continued infection of $\mathrm{CD}^{+} \mathrm{T}$ lymphocytes. In summary, SLBP links the processes of inflammation, histone metabolism, and HIV-1-centric chromatin accessibility and operates in vivo as an HIV-1-related host regulatory factor.

\section{Methods}

Supplemental Methods are available online with this article; doi:10.1172/JCI82360DS1.

\section{Labeling of cells}

Five million peripheral blood mononuclear cells (PBMCs) from each subject with VL greater than 10,000 copies/ml were pooled and then labeled as VL ${ }^{\text {high }}$. Cells were cultured in SILAC RPMI-1640 labeling medium, designated as "heavy" medium (containing 10\% dialyzed FBS and supplemented with $100 \mathrm{mg} / \mathrm{l}$ [U-13C $\left.\mathrm{C}_{6}\right]$-L-lysine, $100 \mathrm{mg} / \mathrm{l}$ [U-13C $6 \mathrm{C}_{6}$ ]-L-arginine, and $1 \mathrm{X}$ L-glutamine; Thermo Fisher Scientific). Similarly, 5 million PBMCs from each subject with VL less than 2,000 copies/ml were also pooled and then labeled as VL ${ }^{\text {low }}$. These cells were maintained in RPMI-1640 medium (Thermo Fisher Scientific), designated as "light" medium (containing 10\% dialyzed FBS and supplemented with $100 \mathrm{mg} / \mathrm{l} \mathrm{L}$-lysine, $100 \mathrm{mg} / \mathrm{l} \mathrm{L}$-arginine,

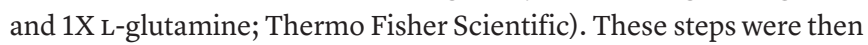
repeated using a second group of pooled samples from different individuals with comparable high and low viral loads.

\section{Protein extraction and preparation}

Expanded "heavy" and "light" cells were each spun down at $200 \mathrm{~g}$ for 10 minutes. Cells were washed once with cold PBS before mixing with CelLytic M reagent (MilliporeSigma) for 15 minutes on a shaker. The supernatant containing total protein extract was collected after 10 minutes of centrifugation at $12,800 \mathrm{~g}$. Protein concentrations from both "heavy" and "light" cells were determined by Micro BCA Protein Assay Kit (Thermo Fisher Scientific). "Heavy" $(2 \mu \mathrm{g})$ and "light" $(2 \mu \mathrm{g})$ protein samples were mixed 1:1 before running on NuPAGE $4 \%-12 \%$ Bis-Tris Precast Gel (Thermo Fisher Scientific) using an XCell SureLock Mini-Cell (Thermo Fisher Scientific). The gel was stained by SimplyBlue SafeStain (Thermo Fisher Scientific). The entire sample lane from the destained gel was cut into 15 equal gel pieces for fractionation. Each piece was washed 3 times with $50 \%$ acetonitrile/50\% HPLC-grade water before mass spectrometric analysis.

\section{Mass spectrometry and quantitative analysis}

Gel pieces were washed, reduced with DTT, and alkylated with iodoacetamide. Gels were then digested with modified trypsin (Promega) at $\mathrm{pH} 8.3$ overnight at $37^{\circ} \mathrm{C}$ with shaking. The resulting peptide mixtures from each gel piece were analyzed separately by data-dependent microcapillary reversed-phase liquid chromatography tandem mass spectrometry (LC-MS/MS). LC-MS/MS was performed using an EASY-nLC nanoflow HPLC (Thermo Fisher Scientific) with a selfpacked $75 \mu \mathrm{m}$ ID $\times 15 \mathrm{~cm} \mathrm{C}_{18}$ column in buffer A ( $0.1 \%$ formic acid in water) coupled to an LTQ-Orbitrap XL mass spectrometer (Thermo Fisher Scientific) using an MS-Fourier Transform target value of $5.0^{5}$ and an MS/MS-Ion Trap target value of 3.0 $0^{4}$ in data-dependent acquisition (DDA)/positive ion mode. The LC gradient was produced over 60 minutes from $2 \%$ B to $38 \%$ B. Buffer B contained acetonitrile. LC/MS-grade solvents were used. The full scan range was acquired between $390 \mathrm{~m} / \mathrm{z}$ and $1,500 \mathrm{~m} / \mathrm{z}$ at 30,000 resolution using a Top5 DDA method via collision-induced dissociation (CID). Precursor ions were excluded for a duration of 2 minutes. Nano-ESI spray voltage of $+3 \mathrm{kV}$ was used without sheath or auxiliary flow gas. The ion count threshold for MS/MS selection was 700 counts. Activation time and $Q$ values were set as default. Generated MS/MS spectra were searched against the reversed and concatenated nonredundant Human International Protein Index database (version 3.68) using Mascot (version 2.2; Matrix Science). Mascot searches used the fixed modification of carbamidomethyl Cys and variable modifications of oxidation of Met and acetylation of protein N-terminus. Two missed cleavages and trypsin with Proline restriction were used during the searches, and a minimum of 6 amino acid residues were allowed. An MS tolerance of $30 \mathrm{ppm}$ and MS/MS tolerance of 0.8 Da were used during Mascot 
searches. The peptide and protein FDR was set at $1 \%$. Protein quantitation was achieved with MaxQuant software (version 1.0.13.13; http://www.maxquant.org) requiring at least 2 peptides per protein (1 razor and 1 unique) for quantitation. Normalized "heavy" /light" $(\mathrm{H} / \mathrm{L})$ ratios were used for all subsequent interpretation.

\section{Bioinformatic analysis}

Protein quantitation. Two prerequisites were applied to ensure greater confidence in the accuracy of the MS-derived peptide ratios: (a) Two or more quantifiable peptides were required to be associated with a given protein. (b) In order to determine significantly up- and downregulated protein candidates, conservative cutoff values were calculated as follows: median and SD $(\sigma)$ of normalized H/L ratios were calculated using log-transformed data of all quantified proteins. The cutoff of value (median $\pm 2 \sigma$ ) was calculated in log space that was then transformed back into linear space. All identification and quantitation were also manually inspected using previously published methodologies (56-58).

Histogram of $H / L$ ratio distribution. Normalized $\mathrm{H} / \mathrm{L}$ ratios of all quantified proteins were $\log _{2}$ transformed, and plotted using SigmaPlot (version 12.5; Systat Software).

DAVID analysis. To find significantly overrepresented gene ontology terms, Database for Annotation, Visualization and Integrated Discovery (DAVID, version 6.7; http://david.abcc.ncifcrf.gov) analysis was used (59), and functional annotation charts were created with default settings.

$H I V-1 /$ host protein network analysis. The most recent (November 2015) HIV-1/human protein interaction database (http://www.ncbi. nlm.nih.gov/genome/viruses/retroviruses/hiv-1/interactions/) (26) was manually examined to identify species shared by the database and our MS-generated results. The database contains 3,592 human proteins, which have been reported to interact with proteins encoded by HIV-1.

\section{Cell culture, HIV-1 challenge, and p24 quantification}

HeLa-T4 (\#154, NIH AIDS Reagent Program), a human cervical epithelial carcinoma cell line modified to stably express CD4 and CXCR4, was used as a preliminary screening tool. It was maintained in DMEM supplemented with 10\% FBS and antibiotics. CEM cells (CCL-119; American Type Culture Collection [ATCC]), a T lymphoblastoid cell line derived from human leukemia, was used to validate significant findings. It was grown in RPMI-1640 medium with $10 \%$ FBS and antibiotics. Purified CD4 ${ }^{+}$cells (2W-200; Lonza) were also used to verify key findings. They were grown in LGM-3 medium with $10 \%$ FBS (Lonza). All cells were subsequently infected by HIV- $1_{\mathrm{NL} 4-3}$ (starting with $\sim 10 \times 10^{3} \mathrm{TCID}_{50}$ (50\% tissue culture infective dose) per million cells; Malcolm Martin, NIH AIDS Reagent Program) for 48 hours. Infection was monitored by p24 quantification in culture supernatants with Alliance HIV-1 p24 Antigen ELISA kit from PerkinElmer.

\section{siRNAs and cell transfection}

siRNA SMARTpool containing 4 distinct siRNAs against each specific gene (Supplemental Table 8) was purchased from GE Dharmacon. Individual siRNAs of the hits were also obtained for the purpose of verification. Following manufacturer's protocols, transfection was carried out using Lipofectamine RNAiMAX Reagent (Thermo Fisher Scientific) for HeLa-T4 or 4D-Nucleofector (Lonza) for primary CD $4^{+}$ cells. After transfection, cells were incubated in a humidified $37^{\circ} \mathrm{C} / 5 \%$ $\mathrm{CO}_{2}$ incubator for $24-48$ hours before analysis of gene expression.

\section{$\beta$-Gal assay}

$\beta$-Glo Assay System (Promega) was used to quantify $\beta$-gal activity on transfected/infected TZM-bl cells (PTA-5659; ATCC), a HeLa cell line that has integrated luciferase and $\beta$-gal genes under control of the HIV-1 promoter, by following the manufacturer's instructions. Briefly, culture supernatants were removed and $200 \mu 1$ lysis buffer was added to each well and incubated for 5 minutes at room temperature to allow complete cell lysis. One hundred microliters of the cell lysate was then transferred to a white 96-well Costar microwell plate, and a volume of $\beta$-Glo reagent equal to that of the cell lysate was added before measurement of the luminescence using a TopCount NXT Microplate Scintillation and Luminescence Counter (PerkinElmer) and a SpectraMax M5 (Molecular Devices).

\section{Real-time PCR}

Total RNA was extracted using TRIzol (Thermo Fisher Scientific) following standard protocols. The concentrations of all RNA samples were determined by spectrophotometry. An equal amount of total RNA was used for reverse transcription and PCR, which was carried out on a Mastercycler gradient 5331 (Eppendorf) using Maxima First Strand cDNA Synthesis Kit (Thermo Fisher Scientific). Primers were designed using PrimerQuest online tools available at http://www.idtdna.com/ Primerquest/Home/Index. Primer sequences are provided in Supplemental Table 9. Real-time PCR was performed on Mastercycler ep realplex (Eppendorf). All reactions were performed in 96-well plates with the following reagents in a final volume of $20 \mu \mathrm{l}: 1 \mu \mathrm{l}$ of primers $(5 \mu \mathrm{M}$ each for forward and reverse) and 2X Maxima SYBR Green qPCR Master Mix from Thermo Fisher Scientific. Ten nanograms of cDNA was added to this mixture. Triplicate reactions of the target and housekeeping genes were performed simultaneously for each cDNA template analyzed. The PCR reaction consisted of an initial enzyme activation step at $95^{\circ} \mathrm{C}$ for 10 minutes, followed by 40 cycles of $95^{\circ} \mathrm{C}$ for $15 \mathrm{sec}-$ onds and $60^{\circ} \mathrm{C}$ for 1 minute. A threshold cycle $(\mathrm{Ct})$ value was obtained for each sample, and triplicate sample values were averaged. The $2^{-\Delta \Delta \mathrm{Ct}}$ method was used to calculate relative expression of each target gene.

\section{Western blotting}

Cellular protein was harvested, quantified by Micro BCA assay, and run on NuPAGE $4 \%$ to $12 \%$ Bis-Tris Precast Gel (Thermo Fisher Scientific) using an XCell SureLock Mini-Cell (Thermo Fisher Scientific). After electrophoresis, the resolved proteins were transferred onto PVDF membrane using an iBlot Dry Blotting System (Thermo Fisher Scientific). Upon completion of the transfer, membranes were blocked for 1 hour before addition of antibodies to specific proteins (Supplemental Table 10) and incubation overnight at $4^{\circ} \mathrm{C}$. Finally, proteins were detected and analyzed by Odyssey CLx Infrared Imaging System (LI-COR) after incubation with IRDye 680LT- and 800CW-conjugated secondary antibodies for 30 minutes followed by 4 -time PBS with $0.1 \%$ Tween 20 washes.

\section{Quantification of HIV-1 integration by Alu-gag PCR}

DNA was extracted by Genomic DNA Purification Kit (Thermo Fisher Scientific) from HIV-1-infected cells. DNA concentration was determined by SmartSpec Plus (Bio-Rad) spectrophotometer. An equal 
amount of DNA from control and experimental cells was applied to the following 2-step PCR (60): First-round Alu-gag PCR was carried out by mixing of primers (forward: 5'-CCTCCCAAAGTGCTGGGATTACAG-3'; reverse: 5'-GTTCCTGCTATGTCACTTCC-3'), Phusion polymerase (New England Biolabs), and extracted genomic DNAs. Second-round real-time (nested) RU5 PCR was performed by combining of primers (forward: 5'-TTAAGCCTCAATAAAGCTTGCC-3'; reverse: 5'-GTTCGGGCGCCACTGCTAGA-3') and probe (5'-/56-FAM/CCAGAGTCA/ZEN/CACAACAGACGGGCACA/3IABkFQ/-3') with the amplicons from first-round PCR and Luminaris Probe qPCR Master Mixes (Thermo Fisher Scientific).

\section{HIV-1 unspliced transcript quantification}

Aforementioned standard protocols (see "Western blotting" above) were followed for RNA extraction, reverse transcription (cDNA synthesis), and real-time PCR. Unspliced RNA detection was carried out using the following primers (61): forward: 5'-TCAGCCCAGAAGTAATACCCATGT-3'; reverse, 5'-CACTGTGTTTAGCATGGTGTTT-3'); and probes: 5'-/56FAM/ATTATCAGA/ZEN/AGGAGCCACCCCACAAGA/3IABkFQ/-3'.

\section{siRNA-resistant SLBP cDNA design, synthesis, and expression}

The coding sequence of human SLBP (NM_006527.3) was modified by replacement of existing codons with alternative ones without changing of the amino acid sequence. The newly designed SLBP cDNA (Supplemental Figure 10) was synthesized by GenScript and was inserted into pcDNA3.1+N-HA expression vector $\left(\mathrm{SLBP}_{\text {res }}\right)$. The $\mathrm{SLBP}_{\text {res }}$ construct was transfected into cells, and Western blot was used to quantify SLBP expression levels and its resistance to SLBP siRNAs. Parallel experiments using empty control plasmid (provided by Xiaoli Tang, Alpert Medical School of Brown University) were also carried out.

\section{ChIP assay}

ChIP assay was performed using a SimpleChIP Enzymatic Chromatin IP Kit (Magnetic Beads) from Cell Signaling Technology following the manufacturer's protocols. Briefly, HeLa-T4 or CEM cells were treated with $1 \%$ formaldehyde for 10 minutes to cross-link proteins to DNA. The isolated nuclei were digested with micrococcal nuclease and further sonicated to prepare 150- to 900-bp DNA/protein fragments. ChIP-grade anti-SP1 (Abcam) was used to immunoprecipitate SP1 binding fragments. The precipitated chromatin was then treated with proteinase $\mathrm{K}\left(2\right.$ hours at $\left.65^{\circ} \mathrm{C}\right)$ to reverse cross-links. Finally, DNA was purified with attached spin column and subjected to real-time PCR. Normal rabbit IgG (Cell Signaling Technology) was used as a negative control.

\section{EpiQ chromatin analysis}

EpiQ assay was carried out according to the manufacturer's instructions (Bio-Rad). Briefly, cells were spun down and washed once with PBS before resuspension in EpiQ chromatin buffer. Half of the suspension was treated with EpiQ nuclease, while the other half received no treatment. Following digestion, DNA was isolated from both nuclease-treated and -untreated samples with columns supplied in the kit. EpiQ primers specific to the promoter of human HMGA1 and the LTR of HIV-1 were designed according to the supplied Primer Design and qPCR Optimization Guide, per the manufacturer. Real-time PCR was performed on both nuclease-treated and -untreated samples with primers specific to HMGA1 and HIV-1 along with those designed for reference gene $(R H O)$ and control gene $(G A P D H)$. The real-time PCR results were further analyzed by EpiQ Chromatin Kit Data Analysis Tool (version 1.1.18.0915). The final chromatin accessibility values were imported to Heatmap Builder (version 1.0; Stanford) for visualization (62).

\section{TNF- $\alpha$ and SLBP expression analysis in subjects}

Plasma samples from 27 study subjects (based on availability) were used to determine TNF- $\alpha$ expression in HIV-1-infected patients. Among them, 11 study subjects with VL less than 2,000 were assigned to the low-VL group, while 16 subjects with VL greater than 10,000 were assigned to the high-VL group (Supplemental Table 11). TNF- $\alpha$ protein levels were quantified by TNF- $\alpha$ Human Ultrasensitive ELISA Kit (Thermo Fisher Scientific) from $100 \mu$ plasma of each subject. PBMC samples from 90 study subjects were used to determine SLBP expression in vivo. Among them, 21 study subjects with VL less than 2,000 were assigned to the low-VL group, 26 subjects with VL greater than 10,000 were assigned to the high-VL group, 20 subjects under antiretroviral therapy for at least 1 year were assigned to the antiretroviral therapy group, and 23 healthy donors were assigned to the seronegative group (Supplemental Table 12). SLBP protein levels were determined by SLBP ELISA kit (MyBioSource) from protein extracts obtained from 250,000 cells of each subject.

\section{Statistics}

For in vitro samples, all data were based on 3 technical replicates within at least 3 biological replicates. Comparisons between 2 groups were carried out using Student's $t$ test function in SigmaPlot (version 12.5) with 2-tailed distribution. Comparisons among multiple $(\geq 3)$ groups were carried out using 1-way ANOVA with post hoc Tukey's test in Prism (version 6.05; GraphPad). For clinical samples, comparisons were based on at least 3 technical replicates. No statistical method was used to predetermine sample size. The distribution (test of normality) of data in each group was assessed to determine the appropriate use of parametric versus nonparametric tests. Accordingly, Mann-Whitney test was used for comparison of plasma viral load, CD4 count, and plasma TNF- $\alpha$. For analyzing SLBP expression in the 4 groups, 1-way ANOVA with post hoc Tukey's test was used. $P$ value $\leq 0.05$ was considered significant, denoted with an asterisk. $P$ value $\leq 0.01$ is denoted with 2 asterisks. Statistical analyses and graphing were performed with either Prism or SigmaPlot.

\section{Study approval}

This research study was performed under IRB-approved protocols at The Miriam Hospital (Federal Wide Assurance FWA-00003538) and Rhode Island Hospital (Federal Wide Assurance FWA-00001230), both of which are located in Providence, Rhode Island. HIV-1-infected subjects and healthy donors were recruited and enrolled at the Immunology Center at The Miriam Hospital. All enrolled subjects were adults who provided informed consent. Data were collected from participants and their medical records for demographics, medical history, antiretroviral use, and laboratory studies. Deidentified blood samples were obtained from all participants and immediately processed.

\section{Author contributions}

ML designed research studies, conducted experiments, acquired data, analyzed data, and cowrote the manuscript. LDT conducted experiments, acquired data, analyzed data, and pro- 
vided reagents. JMA, CKC, and HL conducted experiments and acquired data. ZJW analyzed data. MCN designed research studies and cowrote the manuscript. MSD conducted experiments and analyzed data. JF supported data analyses. MAL designed research studies and provided reagents. BR designed research studies, analyzed data, and cowrote the manuscript.

\section{Acknowledgments}

This work was supported by an American Recovery and Reinvestment Act (ARRA) supplement to the Lifespan/Tufts/ Brown Center for AIDS Research and by NIH P30-AI04285313S1，P20-RR025179，P20-RR017695，P20-RR017695/P20GM103421, R01-HD072693, and K24-HD080539 to BR. ML was supported by the Lifespan Pilot Research Fund (7015857), a Rhode Island Foundation Medical Research Grant
(20133969), and an NIH COBRE URI/RIH Pilot Research Grant (P20-GM104317) and T32-DA013911. JMA was supported by grant NIH 5P01-CA120964 and NIH DF/HCC Cancer Center Support Grant 5P30-CA006516. We thank Min Yuan and Susanne Breitkopf for help with mass spectrometry experiments and SILAC data processing.

Address correspondence to: Bharat Ramratnam, Department of Medicine, Warren Alpert Medical School of Brown University, 55 Claverick Street, Room 414, Providence, Rhode Island 02903, USA. Phone: 401.793.8905; E-mail: BRamratnam@lifespan.org. Or to: Ming Li, Department of Medicine, Warren Alpert Medical School of Brown University, 55 Claverick Street, Room 432, Providence, Rhode Island 02903, USA. Phone: 401.444.7369; E-mail: ming.li@brown.edu.
1. Cao Y, Qin L, Zhang L, Safrit J, Ho DD. Virologic and immunologic characterization of long-term survivors of human immunodeficiency virus type 1 infection. N Engl J Med. 1995;332(4):201-208.

2. Okulicz JF, et al. Clinical outcomes of elite controllers, viremic controllers, and longterm nonprogressors in the US Department of Defense HIV natural history study. J Infect Dis. 2009;200(11):1714-1723.

3. Samson M, et al. Resistance to HIV-1 infection in caucasian individuals bearing mutant alleles of the CCR-5 chemokine receptor gene. Nature. 1996;382(6593):722-725.

4. Liu R, et al. Homozygous defect in HIV-1 coreceptor accounts for resistance of some multiply-exposed individuals to HIV-1 infection. Cell. 1996;86(3):367-377.

5. Fätkenheuer G, et al. Efficacy of short-term monotherapy with maraviroc, a new CCR5 antagonist, in patients infected with HIV-1. Nat Med. 2005;11(11):1170-1172.

6. International HIV Controllers Study, et al. The major genetic determinants of HIV-1 control affect HLA class I peptide presentation. Science. 2010;330(6010):1551-1557.

7. Fellay J, et al. A whole-genome association study of major determinants for host control of HIV-1. Science. 2007;317(5840):944-947.

8. Pelak K, et al. Host determinants of HIV-1 control in African Americans. JInfect Dis. 2010;201(8):1141-1149.

9. Ranasinghe S, et al. Association of HLA-DRB1-restricted $\mathrm{CD}^{+} \mathrm{T}$ cell responses with HIV immune control. Nat Med. 2013;19(7):930-933.

10. Migueles SA, et al. Lytic granule loading of CD8+ T cells is required for HIV-infected cell elimination associated with immune control. Immunity. 2008;29(6):1009-1021.

11. Sáez-Cirión A, et al. HIV controllers exhibit potent CD8 T cell capacity to suppress HIV infection ex vivo and peculiar cytotoxic T lymphocyte activation phenotype. Proc Natl Acad Sci US A. 2007;104(16):6776-6781.

12. Walker BD, Yu XG. Unravelling the mechanisms of durable control of HIV-1. Nat Rev Immunol. 2013;13(7):487-498.

13. Emu B, et al. HLA class I-restricted T-cell responses may contribute to the control of human immu- nodeficiency virus infection, but such responses are not always necessary for long-term virus control. J Virol. 2008;82(11):5398-5407.

14. Cherepanov P, et al. HIV-1 integrase forms stable tetramers and associates with LEDGF/ p75 protein in human cells. J Biol Chem. 2003;278(1):372-381.

15. Llano M, et al. An essential role for LEDGF/p75 in HIV integration. Science. 2006;314(5798):461-464.

16. Wei P, Garber ME, Fang SM, Fischer WH, Jones KA. A novel CDK9-associated C-type cyclin interacts directly with HIV-1 Tat and mediates its high-affinity, loop-specific binding to TAR RNA. Cell. 1998;92(4):451-462.

17. Price DH. P-TEFb, a cyclin-dependent kinase controlling elongation by RNA polymerase II. Mol Cell Biol. 2000;20(8):2629-2634.

18. Neil SJ, Zang T, Bieniasz PD. Tetherin inhibits retrovirus release and is antagonized by HIV-1 Vpu. Nature. 2008;451(7177):425-430.

19. Brass AL, et al. Identification of host proteins required for HIV infection through a functional genomic screen. Science. 2008;319(5865):921-926.

20. König R, et al. Global analysis of host-pathogen interactions that regulate early-stage HIV-1 replication. Cell. 2008;135(1):49-60.

21. Zhou H, et al. Genome-scale RNAi screen for host factors required for HIV replication. Cell Host Microbe. 2008;4(5):495-504.

22. Bushman FD, et al. Host cell factors in HIV replication: meta-analysis of genome-wide studies. PLoS Pathog. 2009;5(5):e1000437.

23. Jäger S, et al. Global landscape of HIV-human protein complexes. Nature. 2012;481(7381):365-370.

24. Laguette N, et al. SAMHD1 is the dendritic- and myeloid-cell-specific HIV-1 restriction factor counteracted by Vpx. Nature. 2011;474(7353):654-657.

25. Riveira-Muñoz E, et al. Increased expression of SAMHD1 in a subset of HIV-1 elite controllers. JAntimicrob Chemother. 2014;69(11):3057-3060.

26. Fu W, Sanders-Beer BE, Katz KS, Maglott DR, Pruitt KD, Ptak RG. Human immunodeficiency virus type 1 , human protein interaction database at NCBI. Nucleic Acids Res. 2009;37(Database issue):D417-D422.

27. Lahouassa $H$, et al. SAMHD1 restricts the replication of human immunodeficiency virus type 1 by depleting the intracellular pool of deoxynucleoside triphosphates. Nat Immunol. 2012;13(3):223-228.

28. Nitahara-Kasahara Y, et al. Novel nuclear import of Vpr promoted by importin alpha is crucial for human immunodeficiency virus type 1 replication in macrophages. J Virol. 2007;81(10):5284-5293.

29. Pelchen-Matthews A, Giese S, Mlčochová P, Turner J, Marsh M. $\beta 2$ integrin adhesion complexes maintain the integrity of HIV-1 assembly compartments in primary macrophages. Traffic. 2012;13(2):273-291.

30. Barrero CA, et al. HIV-1 Vpr modulates macrophage metabolic pathways: a SILAC-based quantitative analysis. PLoS One. 2013;8(7):e68376.

31. Gadad SS, et al. The multifunctional protein nucleophosmin (NPM1) is a human linker histone H1 chaperone. Biochemistry. 2011;50(14):2780-2789.

32. Deng L, et al. Acetylation of HIV-1 Tat by CBP/ P300 increases transcription of integrated HIV-1 genome and enhances binding to core histones. Virology. 2000;277(2):278-295.

33. Deng L, et al. Enhancement of the p300 HAT activity by HIV-1 Tat on chromatin DNA. Virology. 2001;289(2):312-326.

34. Naji S, et al. Host cell interactome of HIV-1 Rev includes RNA helicases involved in multiple facets of virus production. Mol Cell Proteomics. 2012;11(4):M111.015313.

35. Marzluff WF. Metazoan replication-dependent histone mRNAs: a distinct set of RNA polymerase II transcripts. Curr Opin Cell Biol. 2005;17(3):274-280.

36. Wang ZF, Whitfield ML, Ingledue TC, Dominski $\mathrm{Z}$, Marzluff WF. The protein that binds the $3^{\prime}$ end of histone mRNA: a novel RNA-binding protein required for histone pre-mRNA processing. Genes Dev. 1996;10(23):3028-3040.

37. Sànchez R, Marzluff WF. The stem-loop binding protein is required for efficient translation of histone mRNA in vivo and in vitro. Mol Cell Biol. 2002;22(20):7093-7104.

38. Chen H, Engelman A. The barrier-to-autointegration protein is a host factor for HIV type 1 integration. Proc Natl Acad Sci US A. 1998;95(26):15270-15274.

39. Violot S, et al. The human polycomb group EED protein interacts with the integrase of human immunodeficiency virus type 1 . J Virol. 2003;77(23):12507-12522. 
40. Farnet CM, Bushman FD. HIV-1 cDNA integration: requirement of HMG I(Y) protein for function of preintegration complexes in vitro. Cell. 1997;88(4):483-492.

41. Kalpana GV, Marmon S, Wang W, Crabtree GR, Goff SP. Binding and stimulation of HIV-1 integrase by a human homolog of yeast transcription factor SNF5. Science. 1994;266(5193):2002-2006.

42. Ao Z, et al. Interaction of human immunodeficiency virus type 1 integrase with cellular nuclear import receptor importin 7 and its impact on viral replication. J Biol Chem. 2007;282(18):13456-13467.

43. Larue R, et al. Interaction of the HIV-1 intasome with transportin 3 protein (TNPO3 or TRN-SR2). JBiol Chem. 2012;287(41):34044-34058.

44. Maertens G, et al. LEDGF/p75 is essential for nuclear and chromosomal targeting of HIV-1 integrase in human cells. J Biol Chem. 2003;278(35):33528-33539.

45. Priet S, et al. HIV-1-associated uracil DNA glycosylase activity controls dUTP misincorporation in viral DNA and is essential to the HIV-1 life cycle. Mol Cell. 2005;17(4):479-490.

46. Sadowski I, Lourenco P, Malcolm T. Factors controlling chromatin organization and nucleosome positioning for establishment and maintenance of HIV latency. Curr HIV Res. 2008;6(4):286-295.

47. Archin NM, et al. Administration of vorinostat disrupts HIV-1 latency in patients on antiretroviral therapy. Nature. 2012;487(7408):482-485.
48. International AIDS Society Scientific Working Group on HIV Cure, et al. Towards an HIV cure: a global scientific strategy. Nat Rev Immunol. 2012;12(8):607-614.

49. Schröder AR, Shinn P, Chen H, Berry C, Ecker JR, Bushman F. HIV-1 integration in the human genome favors active genes and local hotspots. Cell. 2002;110(4):521-529.

50. Giresi PG, Kim J, McDaniell RM, Iyer VR, Lieb JD. FAIRE (Formaldehyde-Assisted Isolation of Regulatory Elements) isolates active regulatory elements from human chromatin. Genome Res. 2007;17(6):877-885.

51. Cleynen I, Huysmans C, Sasazuki T, Shirasawa S, Van de Ven W, Peeters K. Transcriptional control of the human high mobility group A1 gene: basal and oncogenic Ras-regulated expression. Cancer Res. 2007;67(10):4620-4629.

52. Jeang KT, Chun R, Lin NH, Gatignol A, Glabe $\mathrm{CG}$, Fan H. In vitro and in vivo binding of human immunodeficiency virus type 1 Tat protein and Sp1 transcription factor. JVirol. 1993;67(10):6224-6233.

53. Wada NI, et al. The effect of HAART-induced HIV suppression on circulating markers of inflammation and immune activation. AIDS. 2015;29(4):463-471.

54. Krishnan S, et al. Evidence for innate immune system activation in HIV type 1-infected elite controllers. J Infect Dis. 2014;209(6):931-939.
55. Nixon B, et al. Genital herpes simplex virus type 2 infection in humanized HIV-transgenic mice triggers HIV shedding and is associated with greater neurological disease. J Infect Dis. 2014;209(4):510-522.

56. Li M, et al. Intercellular transfer of proteins as identified by stable isotope labeling of amino acids in cell culture. J Biol Chem. 2010;285(9):6285-6297.

57. Li M, et al. Quantitative proteomic analysis of exosomes from HIV-1-infected lymphocytic cells. Proteomics. 2012;12(13):2203-2211.

58. Li M, Ramratnam B. Proteomic Characterization of Exosomes from HIV-1-Infected Cells. Methods Mol Biol. 2016;1354:311-326.

59. Huang da W, Sherman BT, Lempicki RA. Systematic and integrative analysis of large gene lists using DAVID bioinformatics resources. Nat Protoc. 2009;4(1):44-57.

60. Liszewski MK, Yu JJ, O'Doherty U. Detecting HIV-1 integration by repetitive-sampling Alu-gag PCR. Methods. 2009;47(4):254-260.

61. Pasternak AO, et al. Highly sensitive methods based on seminested real-time reverse transcription-PCR for quantitation of human immunodeficiency virus type 1 unspliced and multiply spliced RNA and proviral DNA. JClin Microbiol. 2008;46(7):2206-2211.

62. King JY, et al. Pathway analysis of coronary atherosclerosis. Physiol Genomics. 2005;23(1):103-118. 